\title{
Active tactile sampling by an insect in a step-climbing paradigm
}

\author{
André F. Krause ${ }^{1}$ and Volker Dürr ${ }^{1,2 *}$ \\ ${ }^{1}$ Cognitive Interaction Technology - Centre of Excellence, Universität Bielefeld, Bielefeld, Germany \\ 2 Fakultät für Biologie, Lehrstuhl für Biologische Kybernetik, Universität Bielefeld, Bielefeld, Germany
}

\section{Edited by:}

Pavel M. Itskov, Champalimaud

Foundation, Portugal

Reviewed by:

Jeremy E. Niven, University of

Sussex, UK

Jiro Okada, Nagasaki University, Japan

*Correspondence:

Volker Dürr, Fakultät für Biologie, Lehrstuhl für Biologische Kybernetik, Bielefeld University, Universitätsstr. 25, Bielefeld 33615, Germany. e-mail: volker.duerr@uni-bielefeld.de
Many insects actively explore their near-range environment with their antennae. Stick insects (Carausius morosus) rhythmically move their antennae during walking and respond to antennal touch by repetitive tactile sampling of the object. Despite its relevance for spatial orientation, neither the spatial sampling patterns nor the kinematics of antennation behavior in insects are understood. Here we investigate unrestrained bilateral sampling movements during climbing of steps. The main objectives are: (1) How does the antennal contact pattern relate to particular object features? (2) How are the antennal joints coordinated during bilateral tactile sampling? We conducted motion capture experiments on freely climbing insects, using steps of different height. Tactile sampling was analyzed at the level of antennal joint angles. Moreover, we analyzed contact patterns on the surfaces of both the obstacle and the antenna itself. Before the first contact, both antennae move in a broad, mostly elliptical exploratory pattern. After touching the obstacle, the pattern switches to a narrower and faster movement, caused by higher cycle frequencies and lower cycle amplitudes in all joints. Contact events were divided into wall- and edge-contacts. Wall contacts occurred mostly with the distal third of the flagellum, which is flexible, whereas edge contacts often occurred proximally, where the flagellum is stiff. The movement of both antennae was found to be coordinated, exhibiting bilateral coupling of functionally analogous joints [e.g., left head-scape (HS) joint with right scape-pedicel (SP) joint] throughout tactile sampling. In comparison, bilateral coupling between homologous joints (e.g., both HS joints) was significantly weaker. Moreover, inter-joint coupling was significantly weaker during the contact episode than before. In summary, stick insects show contact-induced changes in frequency, amplitude and inter-joint coordination during tactile sampling of climbed obstacles.

Keywords: insect antenna, tactile sense, climbing, inter-joint coordination, active touch, stick insect, Carausius

\section{INTRODUCTION}

The tactile sense provides important sensory cues about the near-range environment, with detailed information about shape, location, and surface properties of touched objects, not all of which is easily accessible to other senses, including surface texture and stiffness. Especially animals that operate under difficult lighting conditions, for example nocturnal or aquatic animals, use their tactile sense to acquire vital information about the surrounding environment. Harbor seals use their whiskers to detect subtle currents of water flow, collecting hydrodynamic information about other aquatic animals (prey, predators, or conspecifics; Dehnhardt et al., 1998; Miersch et al., 2011). Shrews use their vibrissae to catch prey in demanding environments, for example hunting insects in complete darkness or underwater. Their tactile sensors allow them to detect the "gestalt" of objects in a scale- and motion-invariant manner (Anjum et al., 2006; Brecht, 2007). Rats explore their environment by rapidly sweeping their long facial whiskers back and forth. This behavior, called "whisking," is actively controlled relative to the environment and the movement of the animal (e.g., Mitchinson et al.,
2011). By means of whisking, rats can tactually localise objects (Ahissar and Knutsen, 2008) and discriminate fine-scale surface textures (Diamond et al., 2008; Morita et al., 2011).

Elaborate forms of active touch sensing are also found in arthropods (Staudacher et al., 2005). Unlike the mammals mentioned above, arthropods do not sample their environment with patches of specialized hairs, but with a pair of antennae (feelers). Antennae are limbs of the head that, during evolution, have become dedicated sensory organs involved in behavioral functions as diverse as course control, pattern recognition, and tactile localization. For example, lobsters use their antennae for navigation and obstacle avoidance on the ocean floor. They are able to discriminate antennal deflections due to water currents from deflections caused by obstacle contacts (Barnes et al., 2001). Flying insects, for example locusts, sense the airflow by measuring the deflection of their antennae (Gewecke and Heinzel, 1980; Heinzel and Gewecke, 1987). Cockroaches use the passive deflection of their long feelers for behaviors like fast wall tracking (Camhi and Johnson, 1999). During tactile near-range exploration, object localization and pattern recognition are frequently 
observed. Active antennal sensing increases the likelihood of detecting obstacles (Krause and Dürr, 2004). Active sensing is a common strategy among animals for improving information gain (Prescott et al., 2011). Honeybees, for example, are able to discriminate the microtexture of flower petals in the range of few tens of microns (Kevan and Lane, 1985) or artificial gratings (ca. $150 \mu \mathrm{m}$ ) (Erber et al., 1998) by tactile scanning of surfaces with their antennal tip. Bees can also discriminate patterns visually, but on considerably larger scale (Srinivasan, 2010). Cockroaches can distinguish between predators and conspecifics by means of antennation, i.e., repetitive antennal touching (Comer et al., 2003) and show tactually induced turning toward- and climbing of objects (Okada and Toh, 2000, 2006). Stick insects continuously move their antennae during walking and sample the space ahead of the walking direction. Because stick insect antennae are approximately the same length as the front legs, touched obstacles are within reach of the front legs. A re-targeting of ongoing leg swing movements to antennal contact position was described by Schütz and Dürr (2011) and shows that the antennae provide short-latency information for adaptive locomotion. Removing the antennae lowers the animal's climbing performance on square obstacles (Dürr et al., 2003). These examples show the relevance of antennal information for pattern and shape recognition, but also for context-dependent adaptation of motor behaviors. Despite its relevance, neither the spatial sampling patterns nor the kinematics of antennal tactile sampling movements of arthropods are well understood. In particular, studies on shape-dependent differences and touchinduced changes in the movement pattern of antennae are very few. Whereas it is clear that insects use antennal tactile cues for decisions about motor acts dependent on location and distance of touched objects (Bläsing and Cruse, 2004a; Harley et al., 2009), almost nothing is known about timing and coupling of antennal joints during tactile sampling, nor about which part of the antennal flagellum is used for sampling the obstacles in order to acquire the information used for decision-making.

In several stick insect species, the location and orientation of both antennal joint axes are known (Dürr et al., 2001; Mujagic et al., 2007), allowing for inverse kinematic calculation of antennal joint angles in walking and climbing insects (Krause and Dürr, 2004). As in all higher insect orders, the antenna of the stick insect Carausius morosus consists of three functional segments: two short, proximal segments called scape and pedicel, and a long flagellum (Figure 1D). The antenna is articulated by two revolute joints: the head-scape joint (HS joint) and the scape-pedicel joint (SP joint) (Dürr et al., 2001; Staudacher et al., 2005). The long flagellum is almost straight and sufficiently stiff not to bend during self-motion. This simplifies the computation of contact points with obstacles, e.g., by application of intersection algorithms from computer graphics. So far, detailed analysis of antennal inter-joint coordination exist for active tactile exploration during locomotion (Krause et al., 2012) and for antennal sampling of simple objects (Schütz and Dürr, 2011). The latter studies focussed on unilateral antennal movements and on the sampling of a nearly one-dimensional object (vertical rod). Here, we investigate unrestrained bilateral antennal tactile sampling movements in a step-climbing paradigm, involving repeated antennal contacts of both antennae. First, we show the influence of object features on antennal contact patterns and the distribution of contacts along antennal parts, revealing a functional regionalization. Second, we show changes in bilateral antennal coordination, antennal working-range, and cycle frequency prior to and during tactile sampling of the climbed obstacle.

\section{MATERIALS AND METHODS ANIMAL PREPARATION}

All experiments were carried out on adult female stick insects of the species Carausius morosus (de Sinéty, 1901) that were bred in a laboratory culture at Bielefeld University. Five animals were prepared for kinematic analysis by means of markerbased motion-capture. For this, animals were labelled with four custom-made retro-reflective foil markers (Scotchlight M3SS-28 8850, 3M Corp., St. Paul/MN, USA). Markers were placed near the caudal margin of the mesonotum, on the head between the eyes, and on the left and right flagellum of the antenna. The antennal markers were placed on the first third of the flagellum, where the antenna is sufficiently stiff to avoid bending due to the mass of the marker. Markers were fixed to the insect cuticle with a drop of translucent nail polish, except for the antennal markers. The latter were attached by a knot around the flagellum. Markers were approximately $1 \mathrm{~mm}$ in diameter. The neck joint and the joint between the pro- and mesothorax were fixed with hot beeswax, keeping the body axis straight during locomotion and, thus, increasing the accuracy of marker tracking. Fixation of these joints did not impair climbing ability of the animals. It did, however, reduce the turning tendency such that animals were less likely to turn although they were still able to do so.

\section{MOTION CAPTURE}

Animals walked along a $40 \mathrm{~mm}$ wide footbridge while being filmed from above, and via a slanted mirror from the side. Within the field of view of the camera, the footbridge was blocked by a step of 19, 32, 47, or $66 \mathrm{~mm}$ height (Figure 1). Step heights were randomized. Walking trials of five animals were recorded, with at least five trials per animal and step height. Videos were recorded using an infrared-sensitive digital video camera (Basler A602f, Ahrensburg, Germany) equipped with a $70 \mathrm{~mm}$ zoom lens, operated at 100 frames per second. Camera exposure was synchronized with a custom-built infrared flash light. The setup was painted in black and surrounded by black drapery. The room was darkened except for a red light, just bright enough for handling of the insects. The camera was calibrated with the "Caltech Camera Calibration Toolbox for Matlab” (Bouguet, 2005), using Matlab (MathWorks Inc., Natick/MA, USA). Videos were captured via fire-wire (IEEE 1394) by use of custom-written frame grabber software (Christoph Schütz, Bielefeld University) and stored on a standard personal computer. All videos were saved as avi-files and cut using the software VirtualDub (www.VirtualDub.org). Video analysis was implemented in Matlab (VideoTrack M, written by Christoph Schütz, Bielefeld University), based on software as described by Zakotnik et al. (2004). In a first step, potential marker coordinates were detected using a standard image processing sequence (thresholding, dilating, and eroding with 
A

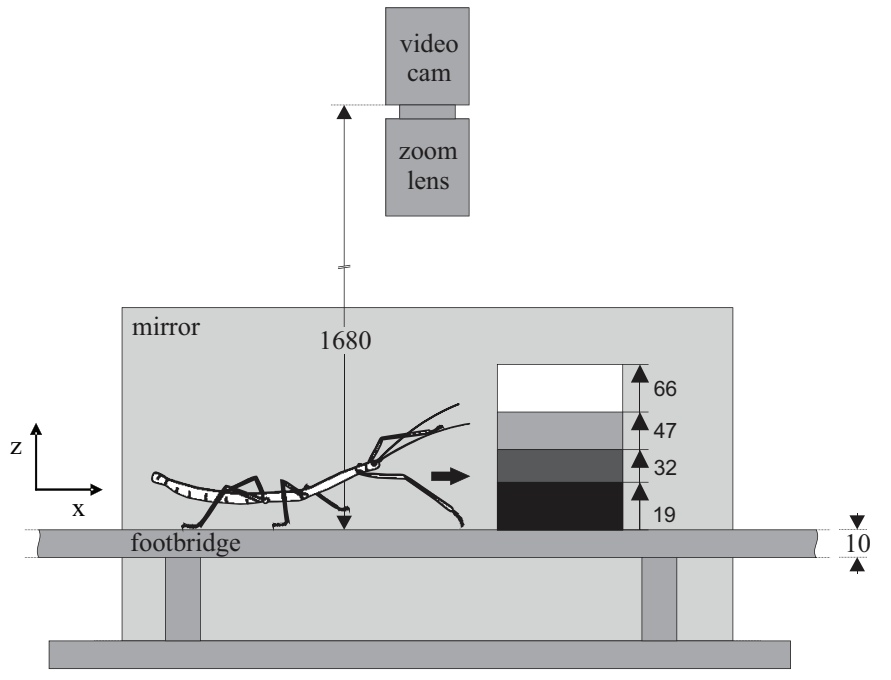

D

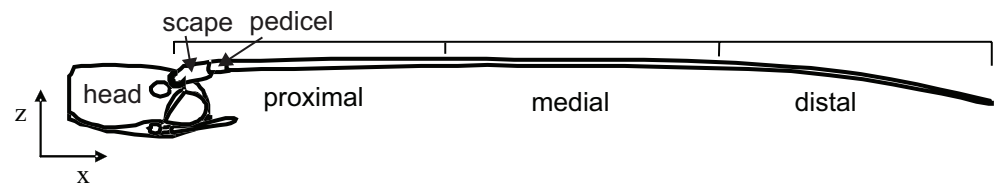

B

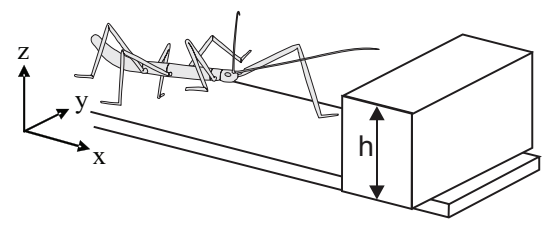

C

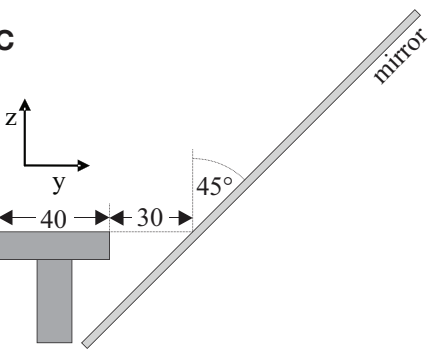

E

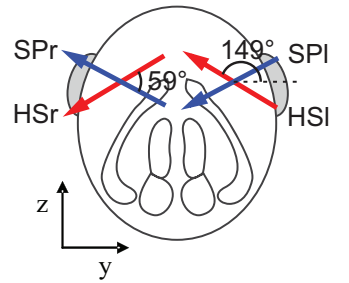

FIGURE 1 | Experimental setup and antennal structure. Stick insects walked freely along a footbridge that was blocked by a step of variable height. Animals were filmed from above, and via a mirror from the side, as they touched and climbed the step. (A) Side view, indicating the four step heights used. (B) Isometric schematic of the setup with steps of variable height, $h$. (C) Front view. The mirror was slanted by $45^{\circ}$ to obtain a side view of the animal. All numbers indicate distances in $\mathrm{mm}$. Coordinate systems indicate the convention used for the coordinate frames. (D) Antennal morphology. Side view of the insect head and right antenna. The first two segments, scape and pedicel, are short and located close to the head, followed by the long flagellum. For later analysis, the antenna was divided into a proximal, medial, and distal section. (E) Antennal kinematics. Schematic front view of the head. The head-scape joint (red arrow) and scape-pedicel joint (blue arrow) are revolute joints. Arrows indicate joint axis orientations relative to the head and are slanted and non-orthogonal. The left head-scape joint (HSI) is almost parallel to the right scape-pedicel joint (SPr), hence these joints are functionally analogous, with rotations in both joints resulting in parallel movement of the left and right antenna. subsequent clustering). Then the root marker of the body model (see below) was marked manually in the first frame and tracked by a nearest-neighbor algorithm.

\section{KINEMATIC ANALYSIS}

Body posture, i.e., segment orientations and antennal joint angles were determined from marker coordinates frame by frame, using a model-based optimization algorithm (Zakotnik et al., 2004) implemented in Matlab (VideoTrack M). Time sequences of coordinates and joint angles were calculated using a Hidden Markov Chain algorithm based on 100 independent optimization runs per frame (Zakotnik and Dürr, 2005). Body models for kinematic analysis were established individually per animal. For this, segment lengths and marker locations along the segments were measured by use of a calliper, achieving an accuracy of at least $0.1 \mathrm{~mm}$. Body models consisted of a kinematic chain including the "body axis" (mesothorax marker to head marker) and an antenna, consisting of the segments scape and pedicel-flagellum. Two such kinematic chains were used for each animal, one for the left and another for the right antenna. As both body models contain the body axis, a discrepancy of the calculated body axis orientation could arise due to independent optimization procedures. A comparison of the pitch and yaw angles of the body axis of each solution served as a measure for the robustness of our motion capture algorithm. Ideally, the differences between the solutions should be zero. Indeed, deviations were always small and the median differences of pitch and yaw angles were well below $1^{\circ}$ (pitch: median $-0.15^{\circ}$, range -0.7 to $0.6^{\circ}$; yaw: median: $0.65^{\circ}$, range -1.4 to $\left.2.3^{\circ}\right)$

The body models contained 6 degree of freedom of rotation per animal and frame: azimuth and elevation of body axis orientation and two joint angles per antenna. Movements of the antennal joints were expressed such that positive angles denote levation above the horizontal plane and negative angles denote depression. Note that the antennal joint axes of stick insects are slanted (Mujagic et al., 2007), such that levation always has a sideward component. Following the notation of Dürr et al. (2001), oblique levation of the SP joint is caused by adductor muscles of the scape and, thus, is a mediad adduction of the pedicel. Similarly, levation of the HS joint is accompanied by a lateral abduction of the scape. Here, antennal joint axes were defined as described in Krause and Dürr (2004). Owing to the mirror symmetry of the head and the slanted antennal joint axes, homologous antennal joints on both sides of the head do not move the antenna 
in the same direction. Rather, it turns out that the left HS joint affects pointing-direction of the left antenna in almost the same way as the right SP joint affects pointing-direction of the right antenna. To emphasize their parallel action, we call bilateral pairs of HS and SP joints "functionally analogous."

Assuming that the flagellum remained almost straight between the antennal joints and the marker, antennal contacts with the obstacle were computed with a ray-box intersection algorithm (Woo, 1990). Note that this procedure neglects curvature of the flagellum in contact with the obstacle and may lead to the situation that an elongate contact zone between the flagellum and an obstacle surface gets reduced to a single point (see Results section on object contacts for further considerations). To compensate for small motion tracking errors, the intersection box was expanded by $1.5 \mathrm{~mm}$. This guaranteed that almost all contacts were identified, as verified by inspection. Contacts with an obstacle, i.e., the step, were distinguished into wall, edge and top contacts, where wall and top contacts correspond to the two surfaces that could be touched. Edge contacts were classified as such, if they were inside a cylindrical bounding region with radius $3 \mathrm{~mm}$ around the edge.

\section{DATA ANALYSIS}

Based on the distinction of antennal contact types, time courses or measured variables were divided into four episodes: an episode before antennal contacts, a wall contact episode beginning with the first wall contact and ending with the first edge contact, an edge contact episode beginning with the first and ending with the last edge contact, and an after-episode beginning with the end of the last edge contact. If both left and right antenna made wall or edge contacts, the average of those contact times was used to delimit the wall and edge episodes. Episodes with less than 25 frames $(250 \mathrm{~ms})$ duration were excluded from further analysis, because a minimum amount of data was required to reliably measure cycle frequency and working-range per episode.

Working-ranges of antennal joints in the four episodes were measured as the 5-95\% quantile ranges of the joint angle distribution. The baseline of a working-range was defined as the $50 \%$ quantile, equivalent to the median joint angle. Cycle frequencies were estimated using FFT, by calculating the weighted average over the amplitude spectrum in the range from 0 to $10 \mathrm{~Hz}$. Patterns of coordination between antennal joints were analyzed in two ways: by cross-correlation of time courses or by a cycleto-cycle phase analysis. Antennal coordination was calculated for six pairs of joints, divided into three groups: ipsilateral (e.g., left HS joint with left SP joint), contralateral (e.g., left HS joint with right HS joint), and functionally analogous joints (e.g., left HS joint with right SP joint). For cycle-to-cycle analysis of phase relationships between antennal joints, we used the Matlab toolkit "Peakfinder" to extract local maxima in the time courses (by Nate Yoder, http://www.mathworks.com/matlabcentral/fileexchange/ 25500-peakfinder). Peakfinder extracts local peaks from noisy data using a threshold, $p$, to determine whether a peak is significantly larger or smaller than the surrounding data. An optimal parameter $(p=8)$ was obtained with the goal to minimize the spread between peak distances. This was based on the assumption of regular periodic antennal movements with almost constant frequency per episode. Only local maxima were extracted, and cycle periods thus concern intervals between two local peak joint angles.

Statistics were calculated with the statistics toolbox of Matlab, and a significance level of $5 \%$ was used throughout this study. Data from $n=5$ animals were used for statistical tests, by averaging extracted parameters, for example antennal working-range, over the five trials per animal. Throughout the text, the following episode pairs were compared for significant differences between kinematic parameters: before vs. wall, wall vs. edge and edge vs. after. In case of multiple comparisons of medians, e.g., in the form of multiple Wilcoxon tests, the significance threshold was Bonferroni-corrected by dividing 0.05 by the number of comparisons (e.g., 0.016 for three comparisons).

\section{RESULTS}

\section{OVERALL OBSERVATIONS}

Stick insects readily walked along the footbridge and climbed the obstacles. As reported in earlier studies (Dürr et al., 2001), all animals showed active tactile exploration behavior by continuously and rhythmically moving both of their antennae during locomotion. Climbing was always accompanied by repeated, bilateral antennation of the obstacle, i.e., tactile sampling with both antennae. Here we report how climbing insects sample the obstacle climbed, and how the antennal movement pattern changes during tactile sampling.

Video-recordings of climbing sequences lasted 2-6s, mainly depending on the height of the obstacle climbed. Four trial conditions were compared, differing in the height of the obstacle, only. The data-set reported in this study comprises a total of 100 trials, with five trials from five animals per condition. All animals used both antennae for tactile sampling with no evidence for a side preference in any of them. Obstacles were sampled on the vertical wall and at the upper edge of the step. Figure 2 shows three representative trials, giving an overall impression of the antennal contact patterns on the obstacle surface, spatial trajectories of both antennal tips, and joint angle time courses of all four antennal joints. In the three trials shown, climbing was accompanied by antennation involving 5, 13, and 20 antennal contacts with the low, middle, and high obstacle, respectively. During tactile contact sequences, the joint angle time courses often revealed marked changes in amplitude and cycle frequency. In the trials shown in Figure 2, these changes are very subtle in the case of the lowest obstacle but obvious in the other two cases, where antennal movement amplitude decreased and cycle frequency increased during tactile sampling. Antennal contacts often occurred during downward antennal movement, i.e., during depression of both antennal joints. In the examples shown in Figure 2, this can be seen by the dashed vertical lines that mark the onset of contact events. In nearly all cases, the antennal joint angle was decreasing when the contact occurred, indicating depression of both scape and pedicel. Note that, in most of the contact events shown, both antennal joint angles reach a local minimum soon after onset of contact, indicating that all antennal joints tended to switch to levation upon tactile contact. This is similar to the situation in unilateral sampling of vertical objects as reported by 

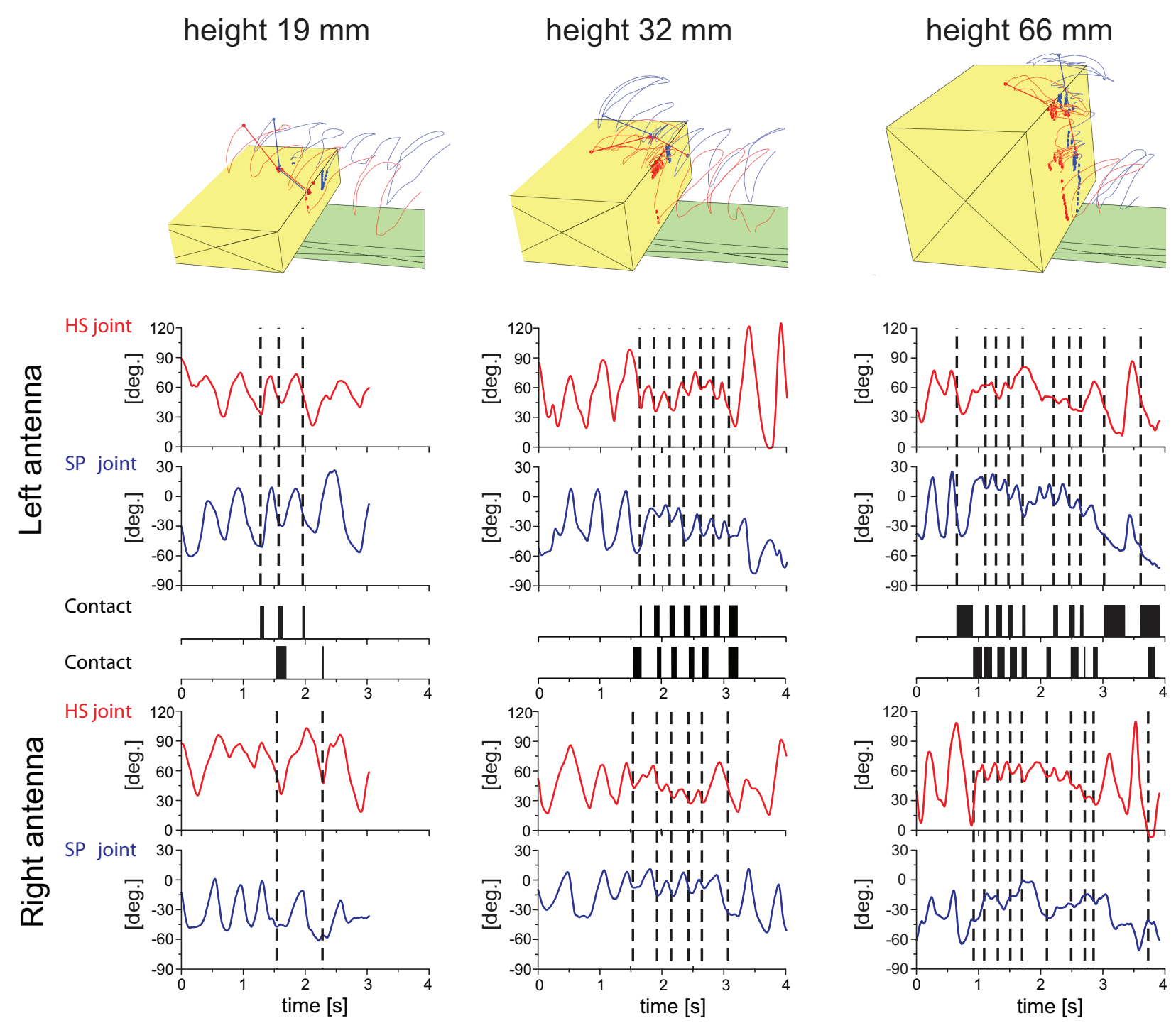

FIGURE 2 | Example trials of stick insects sampling and climbing steps of three different heights. Each column of panels shows data of the same trial. From left to right, step heights were 19, 32, and $66 \mathrm{~mm}$. Top row of panels show schematic reconstructions of the trials as seen from an oblique view. Each panel shows the obstacle (yellow), footbridge (green), and trajectories of the two antennal tips (left: red; right: blue). Antennal contact locations on the obstacle are shown as circles. A stick figure of the body model is drawn for the last frame of the sequence. The six rows of panels below the reconstructions are time courses of the HS joint angle (red lines),
SP joint angle (blue lines) and antennal contact (black rectangles) for the left and right antenna. Vertical dashed lines mark the onset of a contact event of the corresponding antenna. The number of contact events, of wall contacts in particular, increased with increasing step height. During tactile sampling, antennal movement amplitude decreased and the cycle frequency increased compared to exploratory antennal movements before the first contact event. Almost all antennal contact events occurred during depression of both antennal joints. Soon after onset of a contact event, both antennal joints tended to switch from depression to levation.
Schütz and Dürr (2011). In 67\% of all trials, we found an intriguing similarity of joint angle time courses between the HS joint on one side with that of the contralateral SP joint during the wall contact episode (criterion: maximum cross-correlation coefficient $>0.5$ within \pm 0.25 s time-lag; see Figure 9). An example for this can be seen in the right trial shown in Figure 2, where the overall structure of the SP joint angle time courses bear less similarity with the joint angle time course of the ipsilateral HS joint than with that of the contralateral HS joint. As the joint axes of contralateral pairs of HS and SP joints are almost parallel, they are essentially functionally analogous joints. Synchronous movement of functionally analogous joints maintains parallel pointing directions of both antennae.

In the following, we analyse these general observations in more detail, beginning with the spatial pattern of contact locations, followed by the changes in amplitude and cycle frequency and by quantification of inter-joint coupling before, during and after tactile sampling. 


\section{CONTACT PATTERNS ON OBSTACLES}

To understand how and where stick insects touched the obstacle during climbing, we estimated antennal contact locations by calculating the intersection point of the vector describing the antennal pointing-direction with the obstacle. If the distance of this intersection point from the antennal base was shorter than the length of the antenna, we assumed that a contact event had occurred. This procedure neglects bending of the antennal flagellum and, as a result, introduces a bias of the estimated location toward the direction of movement. This is because antennal movement will always "drag" the true contact point behind the tangent to the curvature at the antennal base.

Figure 3 shows the distribution of antennal contacts on the surface of the four obstacles used. Individual antennal contact events with a step lasted several frames of the video recording, forming streak-like patterns on the vertical wall of the obstacle. The prevalent direction of the streaks is upward and slightly lateral, i.e., toward the margin of the obstacle. This indicates that the antennae were levated during wall contacts. The lateral component coincides with the movement direction caused by HS

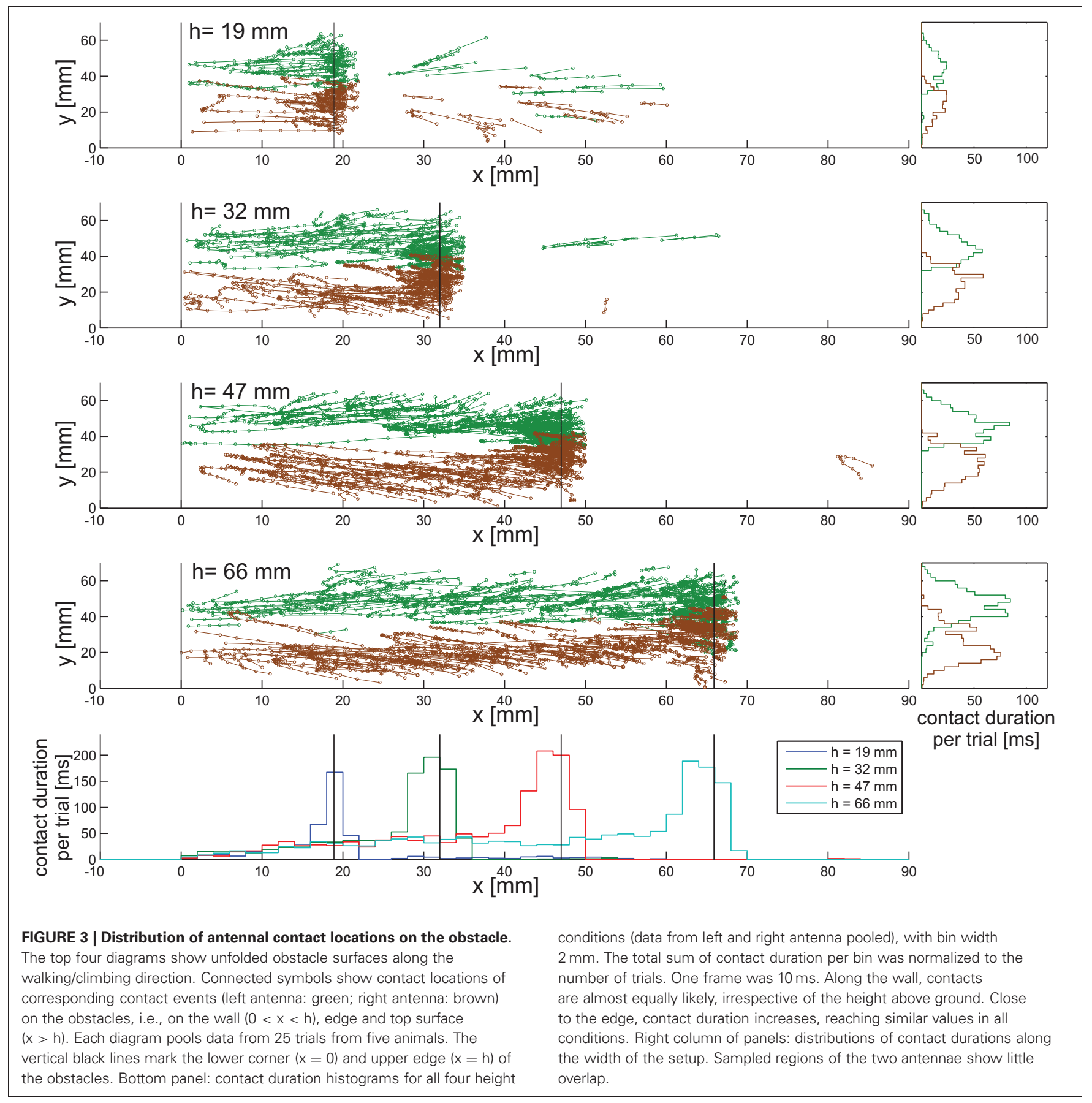


joint levation. In all four conditions, density of contacts increased near the edge of the step, suggesting that the edge was an "interesting feature" to the animal. This is mirrored by the contact duration histograms in Figure 3. All four of these histograms have a broad peak near the edge and a broad, shallow tail at lower contact heights on the wall. The tails indicate that wall contacts are almost equally likely irrespective of height above ground. Close to the edge, contact duration increased to approximately $200 \mathrm{~ms}$ per trial, equivalent to 20 video frames. The apparent lack of contact events on the top surface is partly due to sampling bias, as not all trials had a distinctly long post-contact episode. Contact histograms along the width of the obstacles, i.e., the y-direction, show that both antennae sample different regions with very little overlap. In fact, the contact patterns reveal a region in the middle of the wall that is almost devoid of antennal contacts. This suggests that the overlap of the histograms is mainly caused by edge contacts. Means and standard deviations of the distributions are given in Table 1. Mean locations of left and right

Table 1 | Mean and standard deviation of contact locations along the $\mathrm{y}$-axis in $\mathbf{m m}$.

\begin{tabular}{llllll}
\hline Step & \multicolumn{2}{c}{ Left antenna } & & \multicolumn{2}{c}{ Right antenna } \\
\cline { 2 - 3 } & Mean & SD & & Mean & SD \\
\hline 19 & 44.1 & 8.0 & 24.3 & 7.1 \\
32 & 45.1 & 6.9 & & 24.6 & 7.5 \\
47 & 46.1 & 6.8 & & 24.2 & 7.9 \\
66 & 46.4 & 8.3 & 24.2 & 8.9 \\
\hline
\end{tabular}

antennal contacts were more than two standard deviations apart. More overlap may be expected for animals without fixed neck and prothorax-mesothorax joints.

\section{CONTACT LOCATIONS ON THE ANTENNA REVEAL FUNCTIONAL REGIONALIZATION}

For determining which sensillae were potentially involved in detecting contact events, we were interested to map the distribution of antennal contacts along the antenna. There was a clear difference between the distributions of wall contacts and edge contacts, but also among the wall contact distributions on the four obstacles (Figure 4). Whereas for wall contacts, the distal third of the flagellum spent much more time in contact with the obstacle than the proximal two-thirds, contact durations of edge contacts were nearly equal at all locations along the flagellum (Figure 5B). More than $85 \%$ of wall contact duration involved the distal third of the antenna.

The total duration of wall contacts per trial increased with step height, while the total duration of edge contacts was nearly the same for the three highest obstacles (Figure 5A). Edge contact duration dominated wall contact duration for the two lowest obstacle heights (19 $\mathrm{mm}$ and $32 \mathrm{~mm}$ ). Considering that higher obstacles take longer to be climbed than lower obstacles, we also determined the fraction of the entire tactile sampling episode spent in wall or edge contacts (numbers in Figure 5A). In case of the three highest obstacles, this fraction was 19-23\% for wall contacts, suggesting that the increase in total contact duration was proportional to the increase in duration of the sampling episode (time from onset of first wall contact to end of last edge contact). In case of edge contacts, the fraction decreased with the

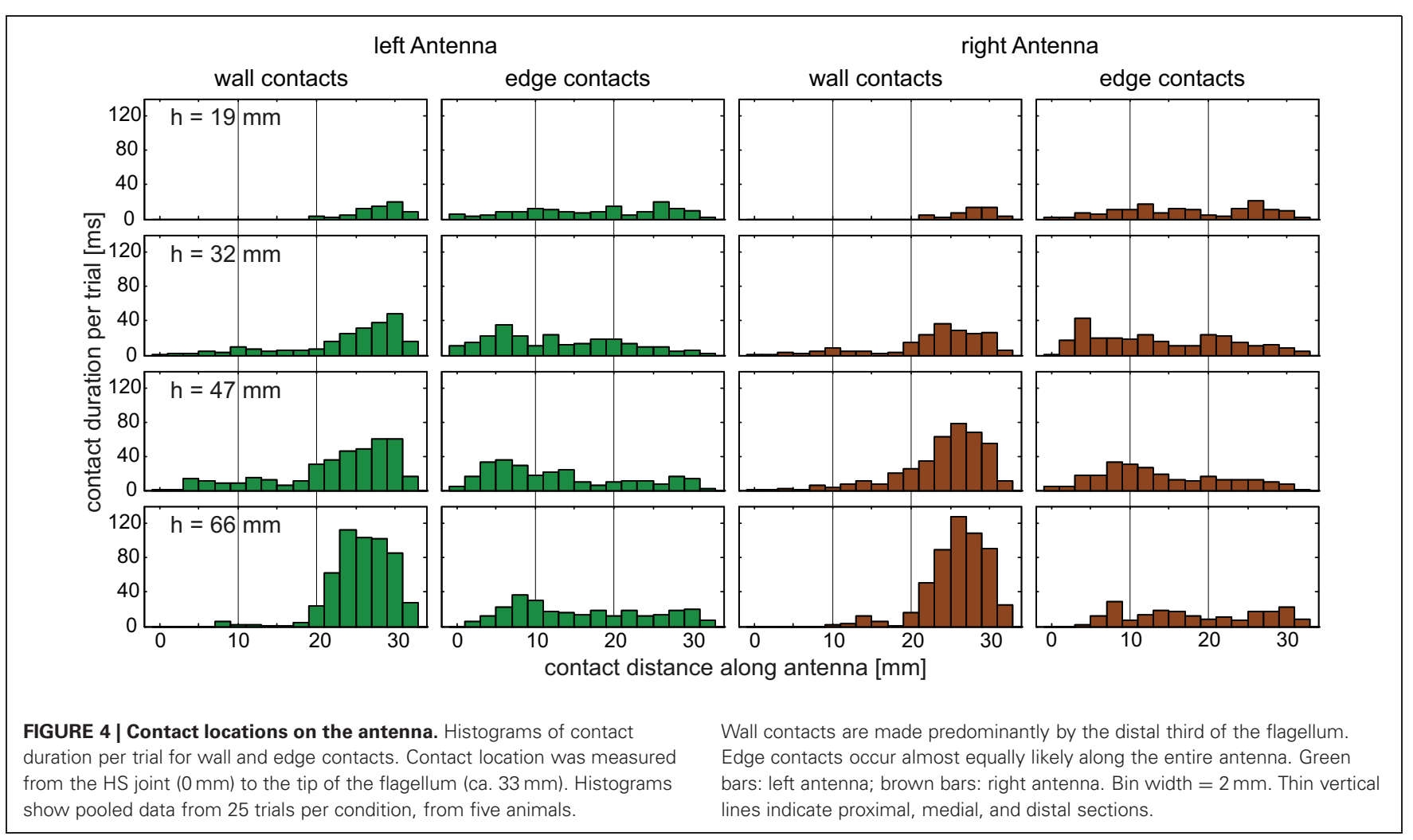




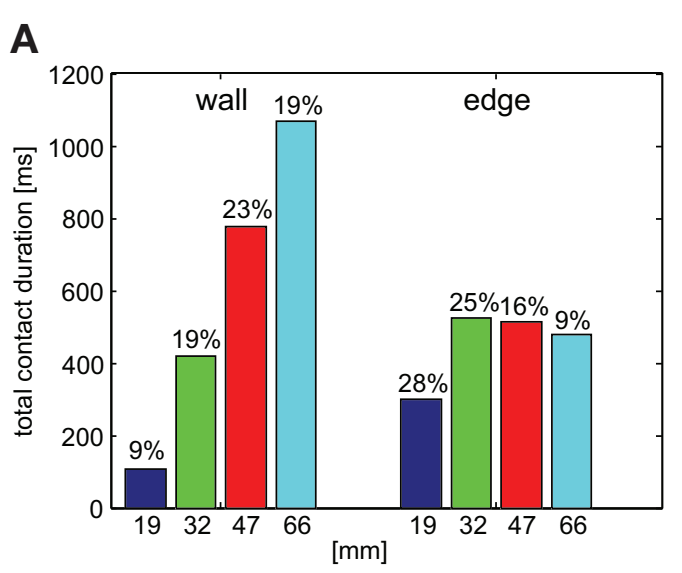

FIGURE 5 | Antennal contact durations. (A) Total contact duration of both antennae per trial, separately for wall and edge contacts. Wall contact duration increases with step height, while edge contact duration is similar for the three highest obstacles. Numbers above bars give the fraction of the total sampling episode with contact events (wall and edge episodes), thus standardizing for
B

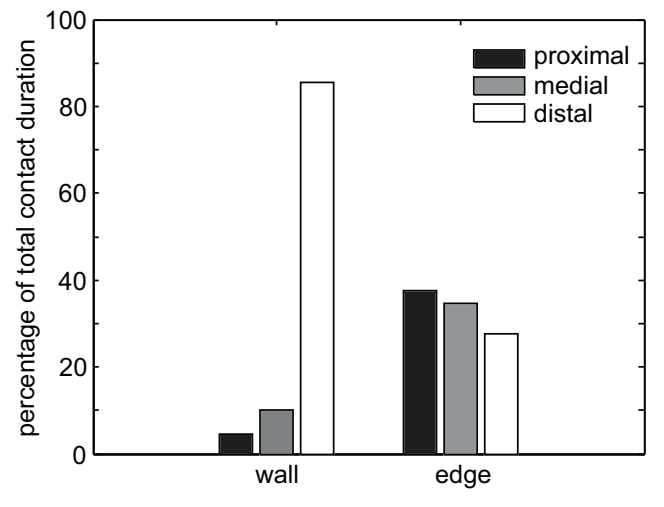

differences in climbing duration. (B) Relative contact duration on three parts of the flagellum (proximal, medial, and distal). Data were separated for wall and edge contacts but pooled across trials and conditions. More than $85 \%$ of wall contacts occurred on the distal part of the flagellum. In contrast, edge contacts were almost equally likely on all flagellum parts. height of the obstacle, as expected if the edge contact episode was equally long but the total sampling episode became longer with obstacle height. Individual contact event durations did not differ significantly between wall and edge episodes, although wall contacts tended to be slightly longer (wall: $134 \pm 82 \mathrm{~ms}$; edge: $115 \pm 84 \mathrm{~ms}$, Wilcoxon's rank sum test on mean values per animal, $n=5, p=0.31$ ).

\section{ANTENNAL WORKING-RANGE AND CYCLE FREQUENCY CHANGE DURING TACTILE SAMPLING}

Next, we tested how the overall kinematics of the antennal movement pattern changed during tactile sampling of the obstacle. To account for potential differences between wall contacts and edge contacts, we determined the antennal working-range and cycle frequency of both antennal joints during four subsequent trial episodes. These were: (1) before first antennal contact, (2) during the wall contact episode, (3) during the edge contact episode, and (4) after the edge contact episode (Figure 6). Note that antennal wall contacts for the lowest $19 \mathrm{~mm}$ obstacle were rare. Only two trials from two animals had wall contacts. While sampling the obstacle wall, the working-range of the HS joint decreased significantly for obstacle heights 32, 47, and $66 \mathrm{~mm}$ (Wilcoxon's rank sum test on mean values per animal, $n=5$, Bonferronicorrected: $p<0.0167)$. A significantly reduced working-range was also found in the SP joint for the $32 \mathrm{~mm}$ obstacle. After the last edge contact, the working-range of the HS joint increased significantly for climbing all but the highest obstacle. In the SP joint, the same effect was statistically significant only for height $47 \mathrm{~mm}$. Additionally, the entire working-range of the antennal joints shifted during climbing, revealing a significant dorsal shift during the wall contact episode in both joints (Figure 8; Wilcoxon's rank sum test on mean values per animal, $n=5$; HS: $10^{\circ}, p=0.016$; SP: $12^{\circ}, p=0.0079$ ).

As antennal working-range decreased, cycle frequency tended to increase in both joints, though this effect was statistically significant only for obstacle heights 32 and $47 \mathrm{~mm}$. Similarly, cycle frequency tended to decrease after the last edge contact, with median values close to those of the pre-contact episode. This effect was statistically significant when climbing low obstacles (HS joint: 19 and $32 \mathrm{~mm}$; SP joint: $19 \mathrm{~mm}$ ). When climbing the highest obstacle, animals on average did not increase cycle frequency, although an increase was evident in some trials (see Figure 2). A possible reason might be that, due to the strong inclination of the body axis, the vertical obstacle wall essentially gets treated like a horizontal surface relative to the animal's body coordinate system. In Figure 6, data from left and right antenna were pooled because no systematic directional bias could be observed. Differences between left and right antennal working-range, base line, and frequency were tested and none of them were significant (Wilcoxon's rank sum test on mean values per animal, $n=5$, $p>0.33)$.

If animals climb obstacles, changes in heading and body axis elevation must be expected. Indeed, apart from changes of the antennal movement pattern, significant changes in body axis orientation were observed (Figure 7). As the neck joint was immobilized in these experiments, body axis orientation was equal to the head orientation and, thus, affected the resting posture of the antennae. The body axis orientation was expressed as yaw and pitch angles, equivalent to polar coordinates (azimuth and elevation, respectively). As expected, the body pitch angle continuously decreased during climbing, and the magnitude of this decrease depended on the height of the obstacle. Significant changes in body pitch angle were found for all obstacle heights but not between all episode pairs (see Figure 7). As the body axis became increasingly inclined, the body yaw angle became more variable: Whereas low steps were climbed with little or no change in yaw angle, its range of variation strongly increased for higher obstacles (height 47 and $66 \mathrm{~mm}$ in Figure 7).

Taken together, the kinematic changes of antennae and body axis are summarized in Figure 8, pooling across all animals 


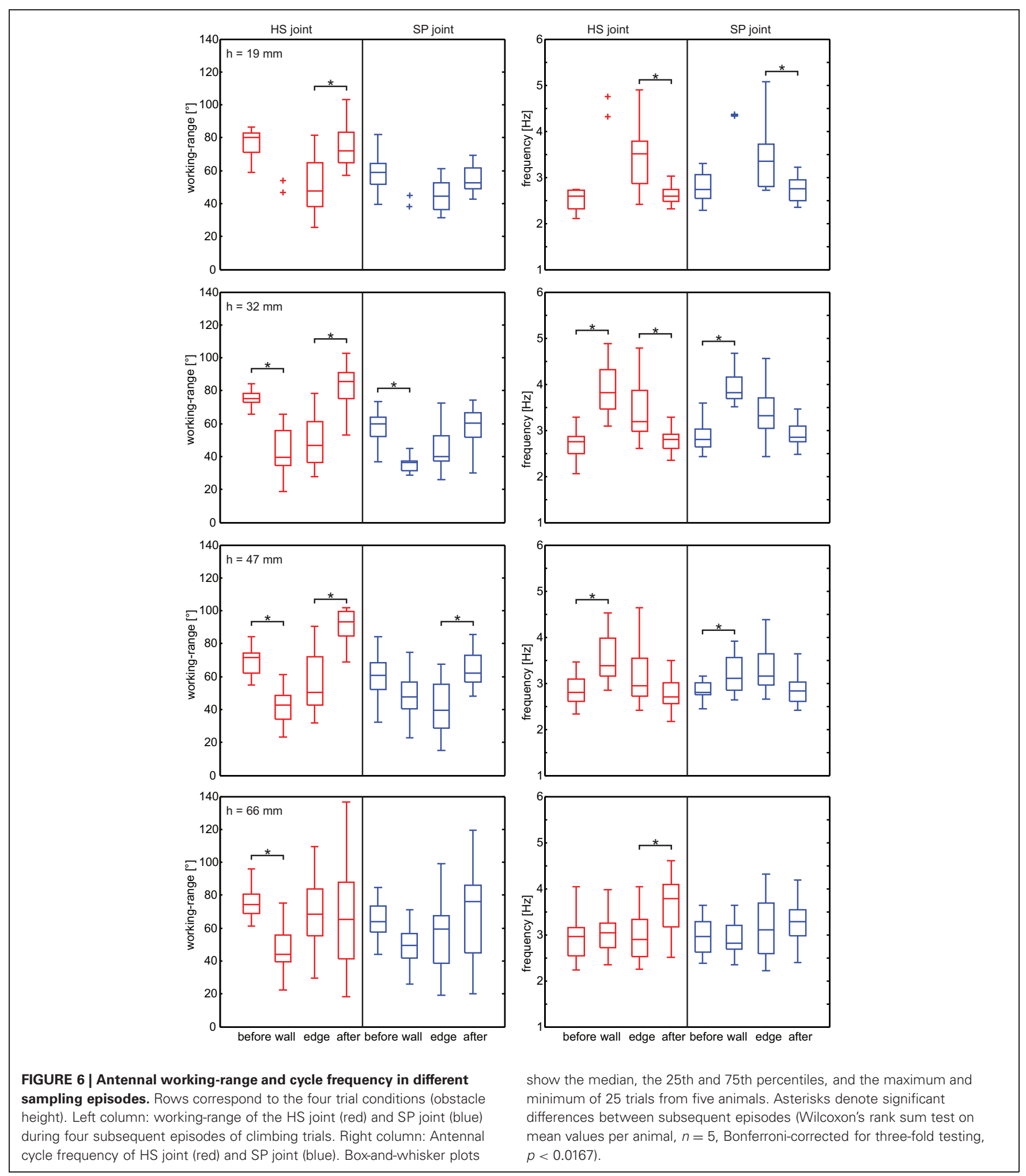

and obstacle heights. During tactile sampling, the joint angle working-ranges significantly shifted upward and decreased in width. At the same time, the cycle frequencies of both joints increased.

\section{ANTENNAL INTER-JOINT COUPLING CHANGES DURING TACTILE} SAMPLING

Finally, we wanted to know whether and how the coordination between antennal joints changed during tactile sampling. For this, 


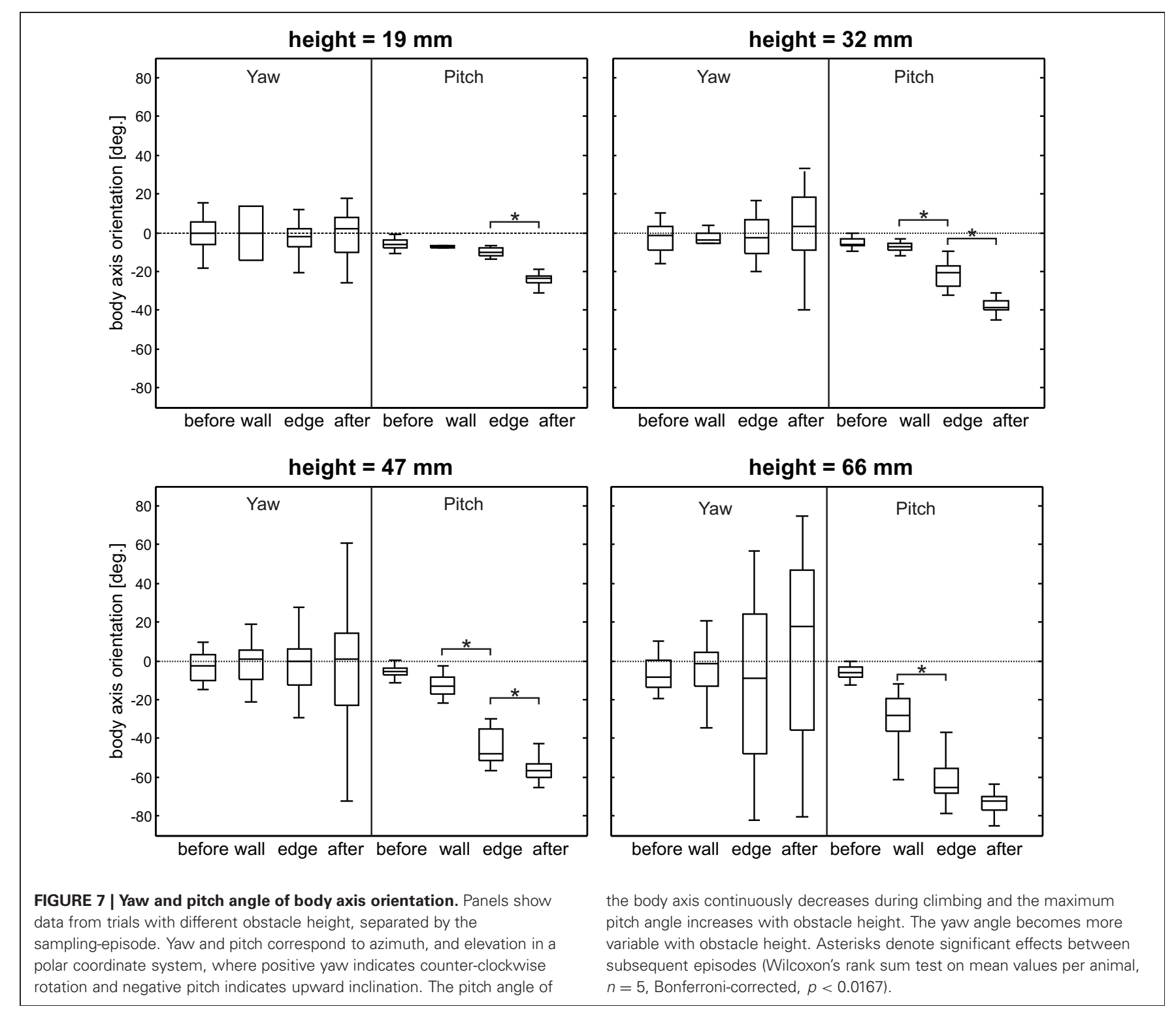

we considered both ipsilateral and contralateral inter-joint coupling, and pairs of antennal joints were grouped into three types: ipsilateral joints (HS-L:SP-L, HS-R:SP-R), contralateral homologous joints (HS-L:HS-R, SP-L:SP-R), and contralateral, functionally analogous joints (HS-L:SP-R, HS-R:SP-L). Functionally analogous joints are joints with nearly parallel joint axes, such that rotation about their joint axis results in nearly the same antennal movement. For example, the left HS joint in C. morosus is almost parallel to the right SP joint (Dürr et al., 2001; Mujagic et al., 2007).

Inter-joint coupling was measured in two ways. The first measure quantified the overall correlation of two joint angle time courses during a certain trial episode. The other measure analysed the cycle-to-cycle phase relationship between the two antennal joints, quantifying the timing of local extrema of their joint angle time courses. Figure 9 shows the distribution of correlation coefficients for the four trial episodes, pooling across all obstacle heights. During the before-contact episode, all types of joint pairs showed correlation coefficients significantly different from zero (Wilcoxon's rank sum test on mean values per animal, $n=5, p=0.0079$ ). During the two sampling episodes with wall and edge contacts, the coordination changed and the median correlation coefficients were close to zero, except for those of contralateral, functionally analogous joints (Wilcoxon's rank sum test on mean values per animal, $n=5, p=0.0079)$. This suggested that functionally analogous joints on both sides of the body midline stay coordinated during tactile sampling.

Whether correlation coefficients close to zero were due to the lack of a fixed coordination pattern or rather due to a $90^{\circ}$ phase shift could not be distinguished without considering non-zero phase lag between the joints. Therefore, Figure 9B shows sliding-window cross-correlograms of a representative trial. For ipsilateral coordination of antennal joints, the correlogram showed a horizontal white band at small negative lag 

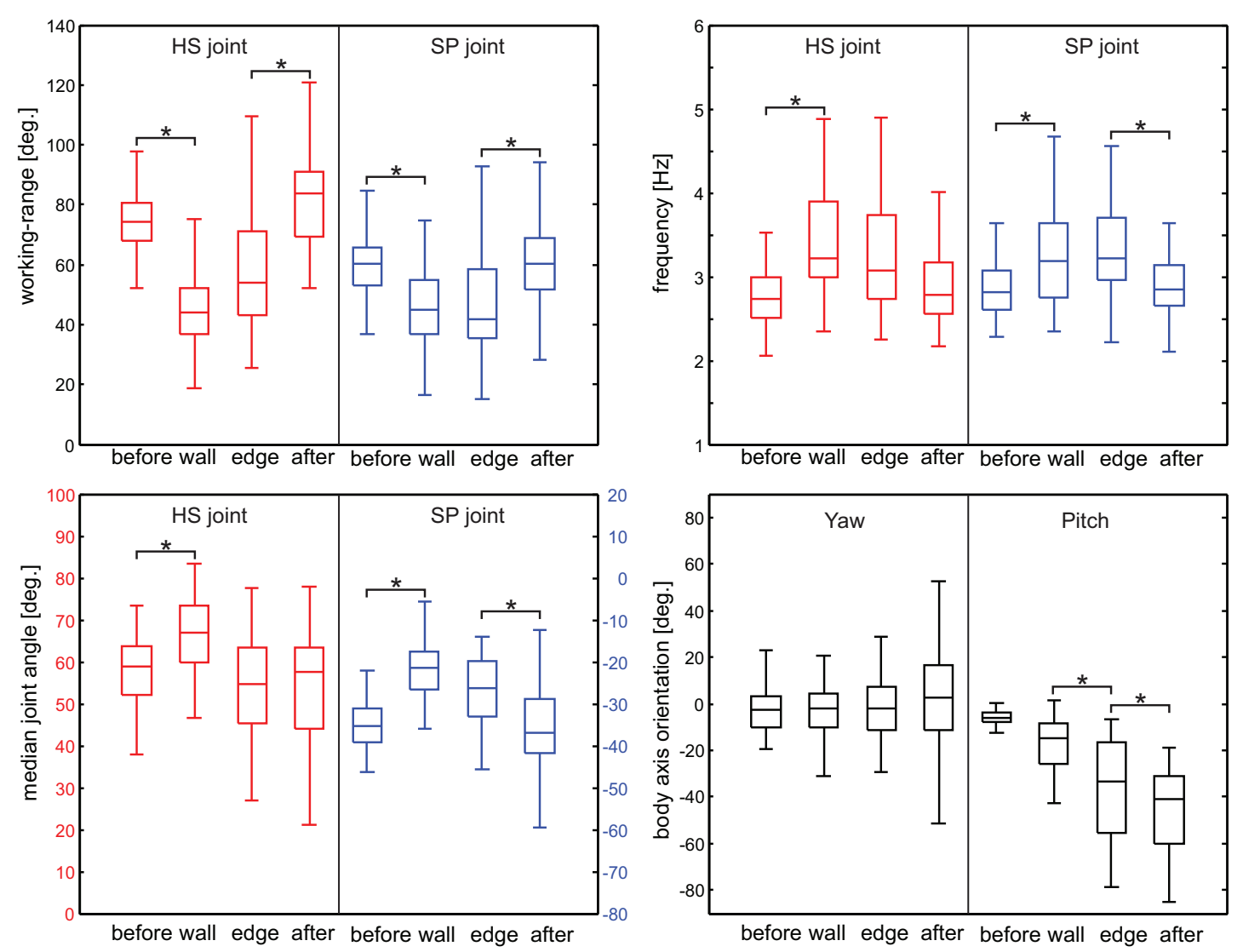

FIGURE 8 | Summary of kinematic changes, pooled across obstacle heights. Top left: Working-range; top right: antennal cycle frequency; bottom left: Center of working-range; bottom right: body axis orientation.

Box-and-whisker plots pool data from 100 trials from five animals and four obstacle heights. Asterisks denote significant effects (Wilcoxon's rank sum

values. This is typical for non-contact episodes of tactile exploration (Krause et al., 2012). This indicated that the alternating movement pattern of the SP joint lead that of the ipsilateral HS joint. During the wall contact episode, the phase between HS and SP joint was less stable, revealing a phase shift compared to the before-contact episode (Figure 9 with a gray to black band where previously was a white band). For contralateral homologous joints the correlogram shows a drifting phase relationship. For contralateral, functionally analogous joints, the initial white band persists well into the tactile sampling episodes, indicating that their coupling changes only little in response to contact events.

The quantitative analysis of cycle-to-cycle inter-joint coupling (Figure 10) revealed similar results as the simple correlation analysis in Figure 9A for contralateral joints (both homologous and functionally analogous joints), and emphasized the role of a systematic phase shift during tactile sampling in case of ipsilateral joints. The phase relationship between peaks revealed consistent and strong coupling between all joint pairs before antennal contact (Rayleigh test, $p<0.0001$ ) with a phase lag between $338^{\circ}$ and $361^{\circ}$ (Figure 10, Table 1). During tactile sampling, cycle-to-cycle phase lag was more variable, test on mean values per animal, $n=5$, Bonferroni-corrected, $p<0.0167$ ). During sampling, antennal working-range decreased and cycle frequency increased in both joints. At the same time, the antennal working-range shifted upward (levation) and the body axis got elevated (negative pitch angle). but was still significant for ipsilateral joints (in both tactile sampling episodes, Rayleigh test, $p<0.01$ ) and contralateral, functionally analogous joints (wall contact episode, Rayleigh test, $p<0.01$ ). In case of contralateral homologous joints, no consistent phase lag could be observed. After the last edge contact, phase relationships tended to return to the values before antennal contact values and showed a significant phase lag in all cases $(p<0.01)$.

If a phase lag in Figure $\mathbf{1 0}$ was found to be statistically significant (Table 2), we tested whether the preferred phase lag changed between subsequent episodes. In the coupling of ipsilateral antennal joints, the phase lag did not change significantly from the before-contact episode $\left(\phi=338.4^{\circ}\right)$ to the wall contact episode ( $\phi=337.6^{\circ}$, Watson-Williams test, $\left.p=0.9427\right)$, but did change significantly from wall to edge contact episode $\left(\phi=375.6^{\circ}\right.$, $p=0.0082)$ and again after the last edge contact $\left(\phi=324.9^{\circ}\right.$, Watson-Williams test, $p<0.0001$ ). For contralateral joint pairs, the phase lag after antennal contact was not significantly different from that before antennal contact in case of homologous HS joints (Watson-Williams test, $p=0.0535$ ) and functionally analogous joints $(p=0.1328)$, but was weakly significant for homologous SP joints (Watson-Williams test, $p=0.0270$ ). 


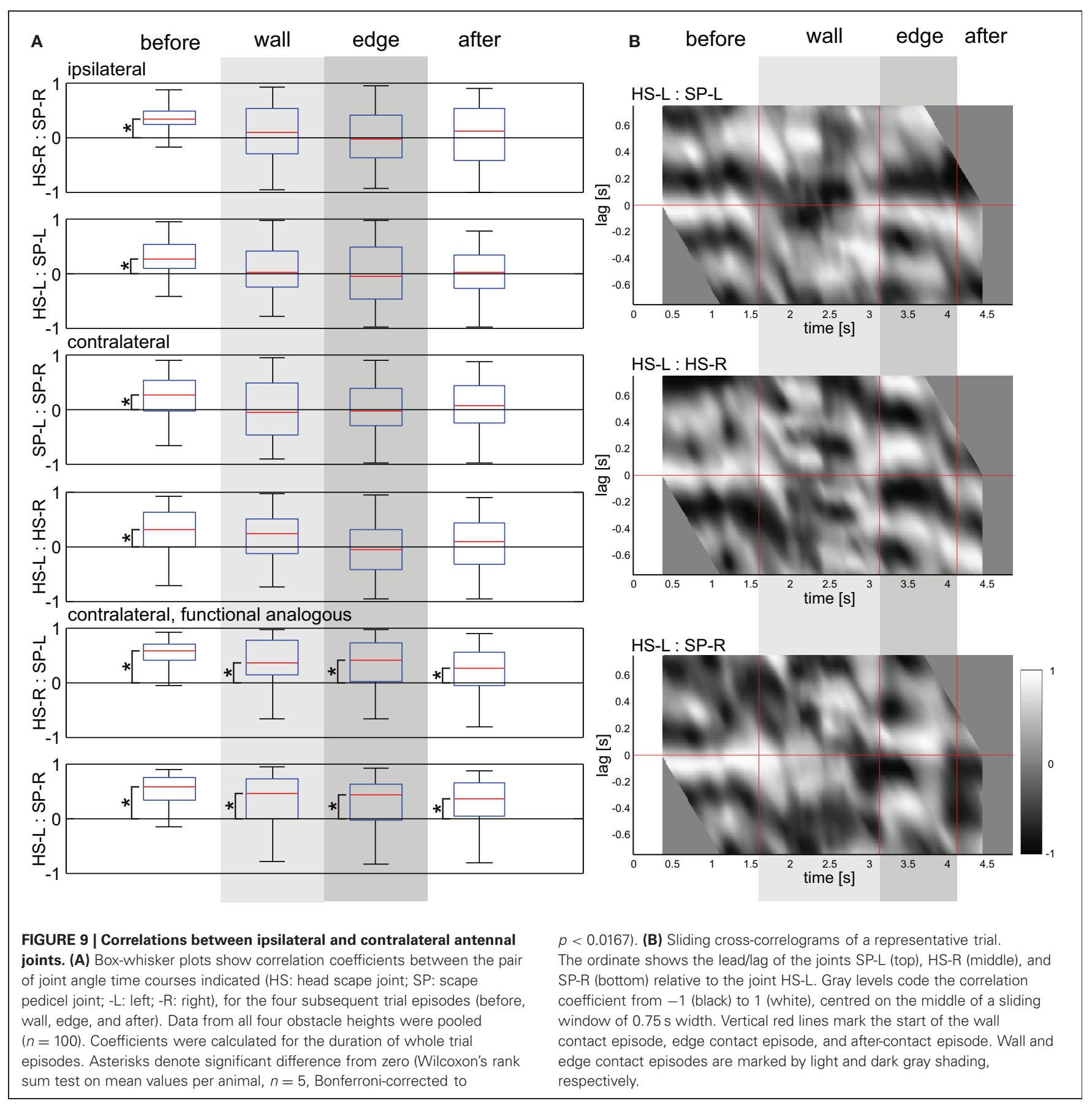

Taken together, antennal inter-joint coupling clearly changed during tactile sampling, though to different degree between different pairs of joints. During active exploration movements without contact events, as in the "before-contact" episode of climbing trials, all antennal joint pairs revealed mutual coupling. Contralateral, homologous joints had an inter-joint coupling with nearly zero phase lag (between -12 and $1^{\circ}$ ), while phase lag between contralateral, functionally analogous joints was very similar to ipsilateral joints $\left(9-20^{\circ}\right)$. During tactile sampling, interjoint coupling generally got weaker (as revealed by lower resultant vector lengths in Table 2) but was still statistically significant between pairs of SP joints and HS joints, though with different phase lag in at least one tactile sampling episode, compared to the episode before contact.

\section{DISCUSSION}

Stick insects use their antennae for near-range exploration (Dürr et al., 2001; Krause et al., 2012) and active tactile sampling of obstacles (Dürr et al., 2003; Schütz and Dürr, 2011). Building on these previous studies on non-contact exploration and unilateral 


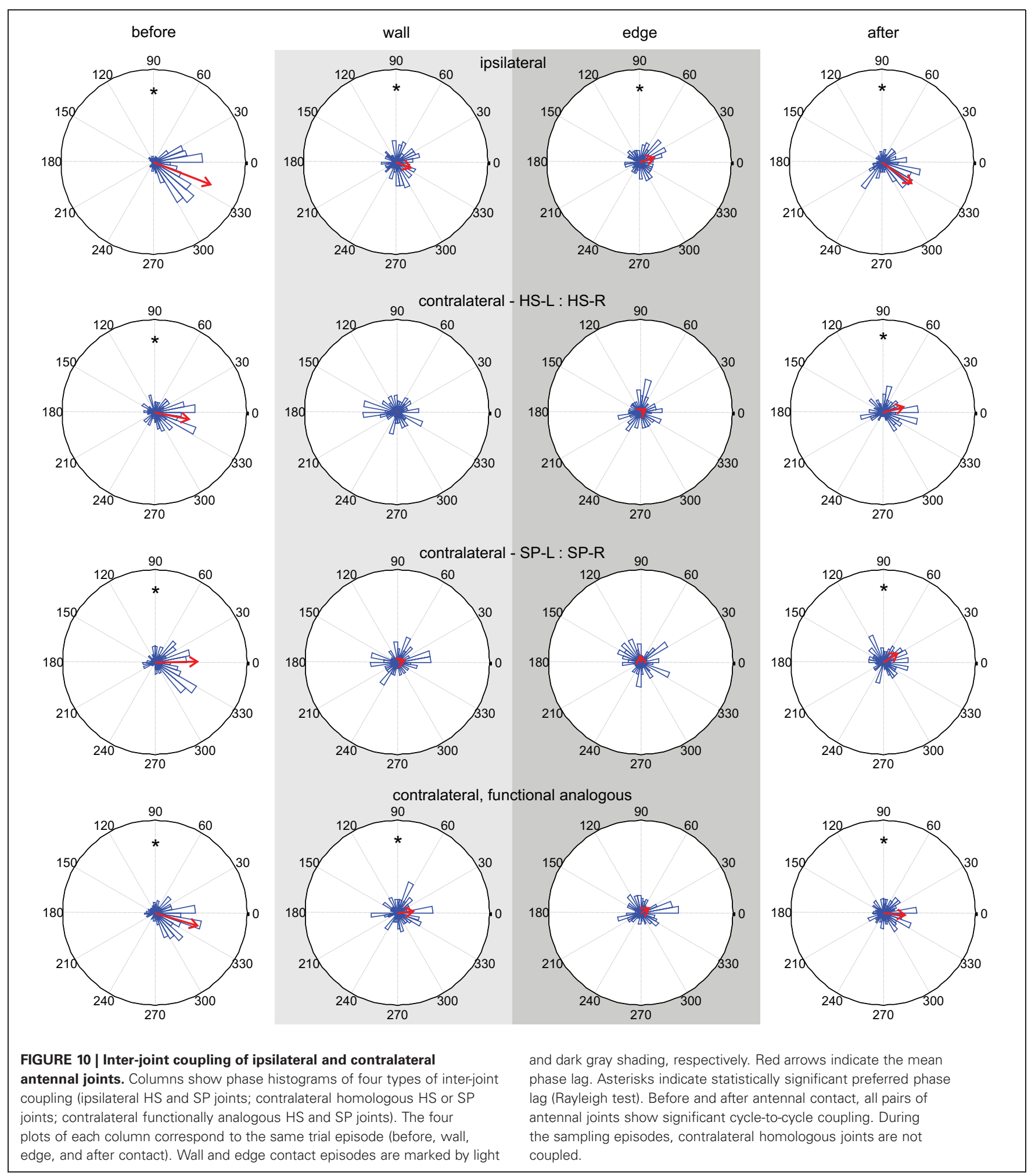

tactile sampling, here we contribute first insights on kinematics and coordination of unrestrained bilateral tactile sampling in a climbing paradigm (Figures 1, 2). We show (1) that the distribution of antennal contact locations differs for distinct object features such as a wall or an edge (Figures 3, 4); (2) that the distribution of contacts along the antenna depends on the contact type, revealing a functional regionalization of the antenna: wall contacts are sampled with the distal part of the flagellum, edge contacts are sampled with the whole flagellum (Figure 4); (3) that antennal working-range and cycle frequency 
Table 2 | Parameters of the phase histograms.

\begin{tabular}{lccl}
\hline & $\phi$ & $\boldsymbol{R}$ & $\boldsymbol{P}$ \\
\hline IPSILATERAL & & & \\
Before & 338.36 & 0.6774 & $0.0000^{*}$ \\
Wall & 337.60 & 0.1932 & $0.0070^{*}$ \\
Edge & 375.57 & 0.1779 & $0.0064^{*}$ \\
After & 324.90 & 0.4137 & $0.0000^{*}$ \\
CONTRALATERAL: $\mathrm{HS}$ & & \\
Before & 348.58 & 0.3977 & $0.0000^{*}$ \\
Wall & 433.72 & 0.0105 & 0.9911 \\
Edge & 387.38 & 0.0801 & 0.5847 \\
After & 373.68 & 0.2486 & $0.0016^{*}$ \\
CONTRALATERAL: SP & & \\
Before & 361.09 & 0.4693 & $0.0000^{*}$ \\
Wall & 377.70 & 0.0896 & 0.5766 \\
Edge & 454.70 & 0.0814 & 0.6096 \\
After & 390.28 & 0.1935 & $0.0084^{*}$ \\
CONTRALATERAL: FUNCTIONALLY ANALOGOUS & \\
Before & 342.47 & 0.4932 & $0.0000^{*}$ \\
Wall & 366.33 & 0.1859 & $0.0072^{*}$ \\
Edge & 392.31 & 0.1152 & 0.1227 \\
After & 355.07 & 0.2501 & $0.0000^{*}$ \\
\hline
\end{tabular}

$\phi$, mean phase; $R$, resultant vector length; $P$, p-values of a Rayleigh test. Asterisks mark rows with p-values below 0.05. Same data as in Figure 10.

is significantly different before, during and after obstacle sampling (Figures 6, 8); (4) that body axis inclination and turning tendency increases with step height (Figures 7, 8); (5) that correlations and phase relationships between ipsilateral, contralateral homologous, and contralateral functionally analogous joints differ between the four trial episodes, revealing a strong coupling between all antennal joints during the pre-contact phase and a weaker coupling of ipsilateral and functionally analogous joints (left HS joint with right SP joint and vice versa) during sampling of the obstacle (Figures 9, 10). Coupling strength of contralateral homologous joints (left and right HS joint, left and right SP joint) reduces during antennation of objects.

Some assumptions were made to simplify the experimental procedure and data analysis. First, possible roll angles around the rostro-caudal body axis were neglected, because it is known that stick insects stabilise their roll angle, keeping it below $10^{\circ}$ even on highly inclined surfaces up to $60^{\circ}$ (Diederich et al., 2002). Second, the stick insect antennae were assumed to be a straight beam for contact point calculation. This neglects the bending of the antennal flagellum during obstacle contacts especially in the highly flexible, distal part of the flagellum. When deflected, the site of maximum curvature of the stick insect flagellum shifts with the contact site (Dürr and Dirks, 2006). This fits well to the finding that the flagellar cuticle gets thicker toward the base (Dirks and Dürr, 2011), suggesting that it is getting stiffer towards the base as well. Thus, edge contacts made with the medial or proximal part of the antenna should cause little antennal deflection. Bending of the flagellum particularly concerns the wall contact events. Owing to the bending, the calculated contact locations on the flagellum must be considered an estimate of the most proximal contact site. The precise location and also the length of the flagellum part that is in contact with the obstacle could not be determined by this study, but it is likely that the contacting region of the flagellum may be several millimetres long, beginning distally from the contact site calculated. As for the contact location on the obstacle, the upward movement of the calculated contact site suggests that the true contact location of the bent distal flagellum is located below, owing to the contacting part being dragged upwards along the contacted wall. As a result, computed wall contact points tend to deviate from the real contact position. Nonetheless, we assume that calculated contact durations are not influenced by antennal bending, because the antenna rapidly returns to its straight posture after object contact (Dirks and Dürr, 2011). Future studies will need to employ a model of antennal bending biomechanics in order to determine contact locations both on the flagellum and on the obstacle with higher precision. Third, prothorax and head movements were suppressed by fixating them with hot bee's wax. This simplified the calculation of the body axis orientation by removing four degrees of freedom from the body model used for marker based motion tracking. These were yaw and pitch rotation of the mesothorax-prothorax joint and of the neck joint. The fixation did not affect locomotion other than making the walking direction more straight. Animals readily climbed obstacles of all heights. Nevertheless, observed body axis elevation values might be different compared to animals with full flexibility in prothorax and head joints.

When walking stick insects encounter an obstacle in their path, they show a strong tendency to climb that obstacle after initial antennal contact (Dürr et al., 2003; Schütz and Dürr, 2011). Antennation continues until the obstacle is climbed, resulting in numerous contacts. Climbing small obstacles caused little change in heading and animals kept almost straight during climbing. In contrast, high obstacles increased the variability of body axis yaw rotation up to $80^{\circ}$. Contact induced change in heading was also reported for cockroaches (Camhi and Johnson, 1999; Okada and Toh, 2000, 2006) and crayfish (Sandeman and Varju, 1988).

Antennal contact information plays an important role for initiation of climbing (Dürr et al., 2003) and body axis inclination increases already after a single antennal contact event (Schütz and Dürr, 2011). During wall- and edge-sampling, animals inclined their body axis, with higher obstacles inducing a more pronounced body axis elevation up to $72^{\circ}$ for the $66 \mathrm{~mm}$ step. Similar results were reported for other insect species. Colorado potato beetles for example raise their body axis proportional to obstacle height after antennal contact to reach the top of the obstacle with the prothoracic leg (Pelletier and McLeod, 1994). Stick insects with cut antennae are less efficient in climbing obstacles (Dürr et al., 2003) or in crossing large gaps (Bläsing and Cruse, 2004b) underlining the importance of tactile information for obstacle negotiation. Cockroaches alter their body posture by first tilting the front of the body upwards and then elevating the center of mass to the height of the step (Watson et al., 2002; Harley et al., 2009). Changes in body posture after wall contacts are also observed in rats. Rats tilt their head upwards as a "contact maximizing" strategy for exploring walls (Grant et al., 2009). 


\section{WHERE DO CONTACT EVENTS OCCUR?}

We show that contact point distribution along the stick insect flagellum differs for contacts on vertical walls and horizontal edges. Stick insects may use the contact distance along the flagellum for behavior adaptation. For example, distal antennal contacts are more likely to occur with a large obstacle (such as a wall) with uncertain height, whereas proximal contacts are more likely to occur with the top edge of an object. While both objects may require climbing, proximal edge contacts contain information about the height to be climbed, whereas distal contacts may require additional sampling for acquiring this information. Comparable results show that cockroaches can maintain a constant distance to a vertical wall using the bend position and contact point along the flagellum in wall-following behavior (Camhi and Johnson, 1999). In their interpretation, flagellar information constitutes a one dimensional sensory map, enabling the control of wall-distance. This interpretation matches the observation of a somatotopic organization of antennal afferents in the deutocerebrum and subesophageal ganglion of the cockroach (Nishino et al., 2005) as well as antennal mechanosensory afferents entering the deutocerebrum of crickets in parallel bands, suggesting a somatotopic map (Staudacher and Schildberger, 1999). We show that left and right antenna of stick insects sample separate "regions of interest" with very little overlap. Mean locations of left and right antennal contacts along the width of the obstacles (y-direction) are more than two standard deviations apart. Broader overlap may be expected for animals without fixed neck and prothorax-mesothorax joints because active head movements may add variability. Separate "regions of interest" per antenna might increase tactile sampling efficacy in terms of overall energy consumption, as was suggested in a previous simulation study (Krause and Dürr, 2004). Assuming dominant viscoelastic friction in joints and muscles, seperate regions of interest reduce overall angular velocity necessary to cover the combined sampling area of left and right antenna and thus reduce energy loss due to velocity dependent friction. Average horizontal edge contact durations $(115 \mathrm{~ms})$ are close to reported contact durations of unilateral sampling of a vertical beam ( $90 \mathrm{~ms}$ ) (Schütz and Dürr, 2011). Mean contact durations seem to be independent of predominant edge or surface orientation of sampled objects, but future studies will need to clarify the relationship between object properties and contact durations in stick insects.

\section{TACTUALLY INDUCED CHANGES IN KINEMATICS AND INTER-JOINT COUPLING}

While sampling the wall of an obstacle, stick insects show significantly higher antennal cycle frequencies in both the HS and SP joint. In combination with a significantly reduced workingrange in both joints a contextual switch after first antennal contact from a broad, exploratory search pattern to a fine-grained object sampling pattern was observed. Schütz and Dürr (2011) reported a four-fold increase in antennal cycle frequency after first contact with a vertical beam. In this study, cycle frequency increase is not as pronounced. Reasons can be the different behavioural context (step with vertical face and horizontal edge vs. vertical beam) and different analysis methods. Here, cycle frequency was estimated from a weighted average of the FFT spectrum in contrast to frequency estimation by counting the number of local minima and maxima in Schütz and Dürr (2011). Context- modulated changes in antennal behavior were reported in Okada and Toh (2006) for cockroaches, where contact frequency positively correlates with the animals turn angle toward a wooden rod placed at a lateral position relative to the animal. Rats show a very similar behavior: in "exploratory whisking," they sweep their whiskers in large amplitudes with a frequency in a range of $5-15 \mathrm{~Hz}$. If presented an object, rats thrust their whiskers forward and palpate the object with low-amplitude and high frequency $(15-25 \mathrm{~Hz})$ movements called "foveal whisking" (Berg and Kleinfeld, 2003). Additionally, rats reduce the "spread" between individual whiskers to maximize surface contacts while sampling vertical walls (Grant et al., 2009). Other small mammals also show contact induced changes in whisking patterns (Brecht et al., 2011; Mitchinson et al., 2011). An interesting question is the role of antennal proprioceptors for the control of active tactile sampling. Okada and Toh (2000) have shown the importance of HS joint hair plates for orientation toward touched objects in cockroaches. Krause et al. (2012) have shown the influence of antennal hair fields on antennal working-range during exploratory, non-contact antennal movements in stick insects. Future studies will need to investigate the effect of antennal proprioceptor ablation on antennal coordination, kinematic parameters, and contact patterns in the stick insect.

Little is known about the temporal coordination of antennal movements on the level of individual joints in insects. Okada and Toh (2004) found that bilateral antennal coordination depends on the animal's behavioral state and is significantly stronger in walking than in resting. Schütz and Dürr (2011) reported a distinct switch in ipsilateral antennal coordination while unilateral sampling of vertical rods. Ipsilateral antennal joint coordination in stick insects was reported to be strong in non-contact situations and was not affected by ablation of proprioceptive hair fields close to the antennal joints (Krause et al., 2012). Apart than that, a simulation study suggested that an elliptical sampling pattern with a fixed phase relationship in ipsilateral antennal joints increases the likelihood to detect obstacles (Krause and Dürr, 2004). In this study we show that antennal inter-joint coordination changes upon obstacle contact. In the pre-contact episode, we observe a strong cycle-to-cycle coupling among ipsilateral, contralateral and functionally analogous joints. After antennal contact, ipsilateral cycle-to-cycle coordination is weaker, but still significant with a distinct change in mean phase while edge-sampling, indicating a contact-triggered switch in sampling behaviour (Figure 10, Table 2). Bilateral antennal coordination changes upon obstacle contact. Correlation values in Figure 9 indicate a clear difference between contralateral homologous and contralateral functionally analogous joints. Functionally analogous joints keep a weak but significant cycleto-cycle coupling during wall sampling (Figure 10), resulting in concerted parallel movements of both antennae. This might be a special characteristic of stick insect antennae or could be an efficient bilateral obstacle sampling strategy. Homologous joints in contrast show no consistent phase lag anymore during contact episodes. 
During obstacle sampling, both antennae now seem to operate more contact-feedback driven and independent from each other. After tactual contacts (post-contact episode), bilateral phase coupling tends to return to pre-contact values. Post-contact episodes in this set of experiments were rather short, because high speed recording time was limited to RAM size. A full recovery to precontact coordination and phase relationships after an obstacle was completely climbed is predicted. Contact driven feedback control is discussed by Mitchinson et al. (2007) for rats. They found that whisker movements are under active, contact induced feedback control to increase the likelihood of environmental contacts.
The presented obstacle climbing paradigm revealed context dependent and contact induced behavior adaptation of active antennal movements in the stick insect Carausius morosus, gaining further insights into tactile object sampling strategies using long, thin probes.

\section{ACKNOWLEDGMENTS}

This work was supported by DFG grant DU380/3 and EU grant EMICAB (FP7-ICT, grant no. 270182), both to Volker Dürr. We thank Leslie Theunissen for helpful comments on earlier versions of the manuscript.

\section{REFERENCES}

Ahissar, E., and Knutsen, P. M. (2008). Object localization with whiskers. Biol. Cybern. 98, 449-458.

Anjum, F., Turni, H., Mulder, P. G., van der Burg, J., and Brecht, M. (2006). Tactile guidance of prey capture in Etruscan shrews. Proc. Natl. Acad. Sci. U.S.A. 103, 16544-16549.

Barnes, T. G., Truong, T. Q., Adams, G. G., and McGruer, N. E. (2001). Large deflection analysis of a biomimetic lobster antenna due to contact and flow. ASME J. Appl. Mech. 68, 948-951.

Berg, R. W., and Kleinfeld, D. (2003). Rhythmic whisking by rat: retraction as well as protraction of the vibrissae is under active muscular control. J. Neurophysiol. 89, 104-117.

Bläsing, B., and Cruse, H. (2004a). Mechanisms of stick insect locomotion in a gap crossing paradigm. J. Comp. Physiol. A Neuroethol. Sens. Neural Behav. Physiol. 190, 173-183.

Bläsing, B., and Cruse, H. (2004b). Stick insect locomotion in a complex environment: climbing over large gaps. J. Exp. Biol. 207, 1273-1286.

Bouguet, J. Y. (2005). Complete camera calibration toolbox for Matlab. Avaliable online at: http://www.vision.caltech.edu/boug uetj/calib-doc

Brecht, M. (2007). Barrel cortex and whisker-mediated behaviors. Curr. Opin. Neurobiol. 17, 408-416.

Brecht, M., Naumann, R., Anjum, F., Wolfe, J., Munz, M., Mende, C., and Roth-Alpermann, C. (2011). The neurobiology of Etruscan shrew active touch. Philos. Trans. R. Soc. Lond. B Biol. Sci. 366, 3026-3036.

Camhi, J. M., and Johnson, E. N. (1999). High-frequency steering maneuvers mediated by tactile cues: antennal wall-following in the cockroach. J. Exp. Biol. 202, 631-643.

Comer, C. M., Parks, L., Halvorsen, M. B., and Breese-Terteling, A. (2003).
The antennal system and cockroach evasive behavior. II. Stimulus identification and localization are separable antennal functions. J. Comp. Physiol. A Neuroethol. Sens. Neural Behav. Physiol. 189, 97-103.

de Sinéty, R. (1901). "Recherches sur la biologie et l' anatomie des Phasmes," La Cellule XIX (J. van In and Cie, Lierre), 118-278.

Dehnhardt, G., Mauck, B., and Bleckmann, H. (1998). Seal whiskers detect water movements. Nature 394, 235-236.

Diamond, M. E., von Heimendahl, M., and Arabzadeh, E. (2008). Whisker-mediated texture discrimination. PLoS Biol. 6:e220. doi: 10.1371/journal.pbio.0060220

Diederich, B., Schumm, M., and Cruse, H. (2002). Stick insects walking along inclined surfaces. Integr. Comp. Biol. 42, 165-173.

Dirks, J. H., and Dürr, V. (2011). Biomechanics of the stick insect antenna: damping properties and structural correlates of the cuticle. J. Mech. Behav. Biomed. Mater. 4, 2031-2042.

Dürr, V., and Dirks, J. H. (2006). "Biomechanics of active tactile sensing with an insect antenna," in Proceedings of the 99th Annual Meeting of the German Zoological Society, (Münster, Germany), 59.

Dürr, V., Krause, A. F., Schmitz, J., and Cruse, H. (2003). Neuroethological concepts and their transfer to walking machines. Int. J. Robotics. Res. 22, 151-167.

Dürr, V., König, Y., and Kittmann, R. (2001). The antennal motor system of the stick insect Carausius morosus: anatomy and antennal movement pattern during walking. J. Comp. Physiol. A 187, 131-144.

Erber, J., Kierzek, S., Sander, E., and Grandy, K. (1998). Tactile learning in the honeybee. J. Comp. Physiol. A 183, 737-744.

Gewecke, M., and Heinzel, H.-G. (1980). Aerodynamic and mechanical properties of the antennae as air-current sense-organs in Locusta migratoria. I. Static characteristics. J. Comp. Physiol. A 139 , 357-366.

Grant, R. A., Mitchinson, B., Fox, C. W., and Prescott, T. J. (2009). Active touch sensing in the rat: anticipatory and regulatory control of whisker movements during surface exploration. J. Neurophysiol. 101, 862-874.

Harley, C. M., English, B. A. and Ritzmann, R. E. (2009). Characterization of obstacle negotiation behaviors in the cockroach, Blaberus discoidalis. J. Exp. Biol. 212, 1463-1476.

Heinzel, H.-G., and Gewecke, M. (1987). Aerodynamic and mechanical properties of the antennae as air-current sense-organs in Locusta migratoria. II. Dynamic characteristics. J. Comp. Physiol. A 161, 671-680.

Kevan, P. G., and Lane, M. A. (1985) Flower petal microtexture is a tactile cue for bees. Proc. Natl. Acad. Sci. U.S.A. 82, 4750-4752.

Krause, A. F., and Dürr, V. (2004). Tactile efficiency of insect antennae with two hinge joints. Biol. Cybern. 91, 168-181.

Krause, A. F., Winkler, A., and Dürr, V. (2012). Central drive and proprioceptive control of antennal movements in the walking stick insect. J. Physiol. Paris (in press).

Miersch, L., Hanke, W., Wieskotten, S., Hanke, F. D., Oeffner, J., Leder, A., Brede, M., Witte, M., and Dehnhardt, G. (2011). Flow sensing by pinniped whiskers. Philos. Trans. R. Soc. Lond. B Biol. Sci. 366 , 3077-3084.

Mitchinson, B., Grant, R. A., Arkley, K. Rankov, V., Perkon, I., and Prescott, T. J. (2011). Active vibrissal sensing in rodents and marsupials. Philos. Trans. R. Soc. Lond. B Biol. Sci. 366, 3037-3048.

Mitchinson, B., Martin, C. J., Grant, R. A., and Prescott, T. J. (2007) Feedback control in active sensing: rat exploratory whisking is modulated by environmental contact. Proc. R. Soc. B Biol. Sci. 274, 1035-1041.

Morita, T., Kang, H., Wolfe, J., Jadhav, S. P., and Feldman, D. E. (2011) Psychometric curve and behavioral strategies for whisker-based texture discrimination in rats. PLoS ONE 6:e20437. doi: 10.1371/ journal.pone.0020437

Mujagic, S., Krause, A. F., and Dürr, V. (2007). Slanted joint axes of the stick insect antenna: an adaptation to tactile acuity. Naturwissenschaften 94, 313-318.

Nishino, H., Nishikawa, M., Yokohari, F., and Mizunami, M. (2005). Dual, multilayered somatosensory maps formed by antennal tactile and contact chemosensory afferents in an insect brain. J. Comp. Neurol. 493, 291-308.

Okada, J., and Toh, Y. (2000). The role of antennal hair plates in objectguided tactile orientation of the cockroach (Periplaneta americana). J. Comp. Physiol. A 186, 849-857.

Okada, J., and Toh, Y. (2004). Spatiotemporal patterns of antennal movements in the searching cockroach. J. Exp. Biol. 207, 3693-3706.

Okada, J., and Toh, Y. (2006). Active tactile sensing for localization of objects by the cockroach antenna. J. Comp. Physiol. A Neuroethol. Sens. Neural Behav. Physiol. 192, 715-726.

Pelletier, Y., and McLeod, C. D. (1994). Obstacle perception by insect antennae during terrestrial locomotion. Physiol. Entomol. 19, 360-362.

Prescott, T. J., Diamond, M. E., and Wing, A. M. (2011). Active touch sensing. Philos. Trans. R. Soc. Lond. B Biol. Sci. 366, 2989-2995.

Sandeman, D. C., and Varju, D. (1988). A behavioral study of tactile localization in the crayfish Cherax destructor. J. Comp. Physiol. A 163, 525-536. 
Schütz, C., and Dürr, V. (2011). Active tactile exploration for adaptive locomotion in the stick insect. Philos. Trans. R. Soc. Lond. B Biol. Sci. 366, 2996-3005.

Srinivasan, M. V. (2010). Honey bees as a model for vision, perception, and cognition. Annu. Rev. Entomol. 55, 267-284.

Staudacher, E., Gebhardt, M. J., and Dürr, V. (2005). Antennal movements and mechanoreception: neurobiology of active tactile sensors. Adv. Insect Physiol. 32, 49-205.

Staudacher, E., and Schildberger, K. (1999). A newly described neuropile in the deutocerebrum of the cricket: antennal afferents and descending interneurons. J. Zool. 102, 212-226.

Watson, J. T., Ritzmann, R. E., Zill, S. N., and Pollack, A. J. (2002). Control of obstacle climbing in the cockroach, Blaberus discoidalis. I. Kinematics. J. Comp. Physiol. A Neuroethol. Sens. Neural Behav. Physiol. 188, 39-53.

Woo, A. (1990). "Fast ray-box intersection," in Graphics Gems, ed A. S. Glassner (San Diego, CA: Academic Press Professional, Inc.), 395.

Zakotnik, J., and Dürr, V. (2005). "Motion analysis using stochastic optimisation and posture disambiguation," in Proceedings of the 3rd International Symposium on Adaptive Motion in Animals and Machines, ed H. Witte (Ilmenau, Germany: AMAM2005).

Zakotnik, J., Matheson, T., and Dürr, V. (2004). A posture optimisation algorithm for model-based motion capture of movement sequences. J. Neurosci. Methods 135, 43-54.

Conflict of Interest Statement: The authors declare that the research was conducted in the absence of any commercial or financial relationships that could be construed as a potential conflict of interest.
Received: 28 March 2012; accepted: 01 June 2012; published online: 28 June 2012.

Citation: Krause AF and Dürr V (2012)

Active tactile sampling by an insect in a step-climbing paradigm. Front. Behav. Neurosci. 6:30. doi: 10.3389/ fnbeh.2012.00030

Copyright (c) 2012 Krause and Dürr. This is an open-access article distributed under the terms of the Creative Commons Attribution Non Commercial License, which permits non-commercial use, distribution, and reproduction in other forums, provided the original authors and source are credited. 